\title{
Validation of the EIKEN Can-do Statements as a Self-assessment Measure Using Rasch Measurement
}

Takanori Sato

Graduate School of Languages and Linguistics of Sophia University

\begin{abstract}
The purpose of the present study was to examine the validity of 16 can-do items taken from the EIKEN can-do list (STEP, 2008). A total of 2,571 Japanese junior high school students were asked to assess their degree of confidence in the 16 can-do statements-four EIKEN Grade 5, Grade 4, Grade 3, and Grade Pre-2 items, respectively. The present study employed the Rasch model to investigate whether (a) the items are unidimensional, (b) their item difficulty is appropriate, (c) item difficulty correlates with the items' EIKEN grades, and (d) the students' confidence levels correlate with their proficiency levels. The results showed that the can-do items are highly reliable and unidimensional. However, the students tended to feel that the items were unchallenging, especially the speaking and listening items.
\end{abstract}

Keywords: validation, can-do statements, self-assessment, Japanese junior high school students, Rasch model, rating scale model

Self-assessment (SA), which is defined as "assessment which involves learners in making judgements about their own level and/or progress" (Davies et al., 1999, p. 177), is widely employed as a measure of L2 learners' language proficiency. In fact, various advantages of incorporating SA are acknowledged in the literature. These include developing linguistic self-awareness (Harmer, 2007) and increasing autonomy and motivation (Brown \& Hudson, 2002). On the other hand, there are some drawbacks, namely, students' subjectivity and inability to discern their errors (Brown \& Abeywickrama, 2010). Despite these disadvantages, SA is used in informal and low-stakes contexts and even in formal and high-stakes situations for placement and research (Oscarson, 1997).

Given the widespread use of SA, investigation of its validity and reliability is appropriate. The present study aims to validate the EIKEN can-do statements developed by the Society for Testing English Proficiency (2008). The validity and reliability of the statements have not been researched extensively. Unlike the past validation studies on SA measures, the present study employed the Rasch rating scale model. 


\section{Literature Review}

\section{The Relationship between SA Ratings and Test Scores}

Two claims have been made regarding SA ratings and test scores: (1) they correlate moderately, and (2) they correlate poorly. Evidence for each of these claims is briefly outlined below.

The studies below indicate that students' assessments of their own language proficiency are moderately accurate. Conducting a meta-analysis of the past research, Blanche and Merino (1989) found that Pearson product-moment correlation values between SA ratings and outcomes of external criteria ranged from .50 to .60 , and that it was not unusual to observe higher values (higher than $r=.70$ ). Another meta-analysis of SA conducted by Falchikov and Boud (1989) showed that the mean value of correlation coefficients between SA and teachers' evaluation was moderate $(r=.39)$. Ross (1998) analyzed correlation for 60 SA measures, concluding that there was a robust correlation on average $(r=.63)$, although the coefficients differed according to the four major skill areas. Several independent studies also indicated a moderate degree of agreement (e.g., Alderson, 2005; Bailey, 1998; Brantmeier \& Vanderplank, 2008).

On the other hand, several studies reported a low correlation and cast doubt on incorporating SA in formal settings. Peirce, Swain, and Hart (1993) found weak correlations (ranging from -.01 to .25) between the SA ratings of students' French proficiency and test scores of French proficiency. Brantmeier (2006) investigated the relationship between SA on reading ability and scores for various reading tasks performed by advanced L2 learners of Spanish. Obtaining a weak correlation between them, the researcher concluded that SA could not accurately predict their performances in computer-based testing and in in-class testing. Finally, Matsuno (2009) discovered that Japanese college students learning English tended to underestimate their abilities, and she advised that SA should not be used for the purpose of formal assessment. There are some causes for the low correlation between self-assessed proficiency and externally measured proficiency. First, the low correlation values are attributable to learners themselves. As reported by Matsuno (2009) and Sullivan and Hall (1997), even advanced learners (college students) occasionally overestimate or underestimate their proficiency to a large extent. The method of measuring self-assessed proficiency can also be responsible. If statements in SA are not closely relevant to the learners' situation or experience, their assessment is often affected (Oscarson, 1997; Ross, 1998).

\section{Approaches to Validation on Self-assessment}

The predominant approach to validating SA measures is to examine concurrent validity, that is, researchers attempt to estimate the correlation between learners' SA ratings and 
their ability in the same domain measured by external resources (e.g., tests, teachers' evaluations). However, this approach has a serious limitation. That is, an external criterion measure is required in order to compare with the SA measure to be validated. Although the external measures can be a well-known standardized test or other measures (Davies et al., 1999), it is not easy to obtain valid and reliable external data, which is an important condition for concurrent validation (Alderson, Clapham, \& Wall, 1995). Strictly speaking, the validity of SA measures cannot be confirmed unless the validity and reliability of test scores or teacher's evaluations are confirmed.

Rasch measurement is another approach to validating SA measures. In fact, there are several applicable Rasch models according to the type of data being investigated, including data from questionnaire surveys. This research employs the rating scale model, which is applicable to questionnaire surveys using the Likert scale. In addition to person and item measures, this model estimates the point where the probability of choosing two adjacent options (e.g., agree and strongly agree) becomes equal, which is referred to as a threshold. An important advantage of using Rasch measurement for validation is that more specific item level analysis is possible than when attempting to demonstrate concurrent validity. In particular, the major concerns when validating a SA measure are whether or not its item difficulty is appropriate to the target students and whether or not all items are measuring a unidimensional construct. Specific item analysis enables item developers to diagnose problematic items and find a solution such as revising or omitting such items.

There are some validation studies on questionnaire items and the Likert response scale that have used Rasch measurement. For example, Mok (2004) investigated self-learning scales that measure students' attitudes toward various self-learning strategies. The difficulty, fit indices, and thresholds of each item were calibrated. Showing the relationship between persons and item thresholds on a single map, Mok (2004) argued that one of the investigated scales needed more difficult items to align properly with the target students. Weaver (2005) also evaluated questionnaire items measuring Japanese college students' willingness to communicate using a similar approach.

\section{The EIKEN Can-do List}

The Society for Testing English Proficiency (STEP) developed and published the EIKEN can-do list (STEP, 2008). These can-do statements are supposedly aligned with each grade level of the EIKEN tests (hereafter EIKEN) and divided into the four skill areas. EIKEN consists of seven grades ranging from Grade 5 (beginner) to Grade 1 (advanced) with two bridging levels (Grade Pre-1 and Pre-2) so test takers can be tested at an appropriate level and receive the result (pass or fail). The Ministry of Education, Culture, Sports, Science and Technology (MEXT) designated Grade 3 as a benchmark for junior high school graduates, and Grade 2 and Pre-2 as a benchmark for 
high school graduates (STEP, 2008).

The EIKEN can-do list was produced on the basis of questionnaire surveys responded to by more than 20,000 EIKEN test takers (Dunlea, 2009; STEP, 2008). Those who had recently passed the exam were given can-do statements with five-point scales and asked what they believed they could do outside a testing situation (e.g., "Can understand simple texts about topics that he/she is interested in."). Consequently, the list shows what a learner is probably capable of doing if he or she passes each EIKEN grade. The can-do list can be employed to help test takers understand specific English abilities targeted by EIKEN, to understand learners' confidence in English use in actual situations, and to do research on English education in Japan (STEP, 2008).

In spite of its research potential, only a few validation studies on the EIKEN can-do statements have been carried out thus far. Takemura (2008) investigated the validity of the writing can-do statements for EIKEN Grade 3 and Grade Pre-2. He developed six writing tasks corresponding to the can-do statements for those levels. A total of 125 college students were asked to indicate their SA on each statement using five-point scales and to perform the developed writing tasks. Comparing both of the results, he found that the learners who gave themselves a high assessment tended to perform the writing tasks more successfully than their peers who gave themselves low ratings.

Similarly, Usuta (2009) examined the validity of the speaking can-do items for EIKEN Grade Pre-2. In his study, 72 college students who passed the EIKEN Grade Pre-2 were asked to engage in four speaking tasks similar to the can-do items. The results showed that more than $80 \%$ of the students were successful in performing three given tasks, although the success rate of another task was $69.4 \%$. Thus, the study concluded that the can-do items were moderately correlated with task performance.

\section{Research Questions}

Given the fact that there are a few studies on the EIKEN can-do statements thus far, it is important to confirm their validity on the basis of empirical data. Therefore, the present study addresses the following research questions:

1. Does each can-do item appear to measure a unidimensional construct?

2. Is the difficulty of the can-do items appropriate for the target students?

3. Do items belonging to the different EIKEN grades (from Grade 5 to Grade Pre-2) reflect students' self-reported confidence levels appropriately?

4. Do the EIKEN can-do items properly reflect the differing confidence levels of students with different proficiency levels? 


\section{Method}

\section{Participants}

In 2007 and 2008, a total of 2,590 junior high school students in Japan participated in a research project on early English language education and responded to a questionnaire survey. This research project was a large-scale survey conducted by the Sophia Linguistic Institute for International Communication (SOLIFIC) and the Japan Institute for Educational Measurement (JIEM). ${ }^{2}$ In 2007, the participants were first- and second-year students studying at five public and private junior high schools and one private English language school. In 2008, second- and third-year students at four public and private junior high schools participated in the survey. Those schools voluntarily cooperated with the survey. The questionnaire, which consisted of questions on the students' attitude toward English learning and EIKEN can-do questions, was distributed to the schools, and the participants were asked to respond to it with teachers' supervision.

Because the original purpose of the survey was to investigate the students' changes or progress over the years, a large number of students overlapped in both surveys, that is, many students responded to the same questionnaire in 2007 and 2008. Since 19 respondents failed to respond to all the EIKEN can-do items in the questionnaire, the responses of 2,571 students were analyzed in the present study. Table 1 presents the number of students in each school year and the EIKEN grades the participants received.

Table 1. The Participants' EIKEN Grades

\begin{tabular}{lllll}
\hline & All & $1^{\text {st }}$ yr. students & $2^{\text {nd }}$ yr. students & $3^{\text {rd }}$ yr. students \\
\hline $\mathrm{N}$ & 2,571 & 694 & 1,365 & 493 \\
Grade 5 & $210(8.2 \%)$ & $76(11.0 \%)$ & $109(8.0 \%)$ & $22(4.5 \%)$ \\
Grade 4 & $474(18.4 \%)$ & $41(5.9 \%)$ & $296(21.7 \%)$ & $137(27.8 \%)$ \\
Grade 3 & $126(4.9 \%)$ & $9(1.3 \%)$ & $55(4.0 \%)$ & $62(12.6 \%)$ \\
Grade Pre-2 & $36(1.4 \%)$ & $5(0.7 \%)$ & $12(0.9 \%)$ & $19(3.9 \%)$ \\
Grade 2 & $10(0.4 \%)$ & $2(0.3 \%)$ & $7(0.5 \%)$ & $1(0.2 \%)$ \\
Grade Pre-1 & $2(0.1 \%)$ & $0(0.0 \%)$ & $2(0.1 \%)$ & $0(0.0 \%)$ \\
Grade 1 & $2(0.1 \%)$ & $0(0.0 \%)$ & $2(0.1 \%)$ & $0(0.0 \%)$ \\
No grade & $1,545(60.1 \%)$ & $507(73.1 \%)$ & $790(57.9 \%)$ & $235(47.7 \%)$ \\
No response & $166(6.5 \%)$ & $54(7.8 \%)$ & $92(6.7 \%)$ & $17(3.4 \%)$ \\
\hline
\end{tabular}

Note. The school year of eighteen respondents was unknown because they did not provide this data. Students who did not have any EIKEN grade were labeled "No grade." 


\section{Can-do Items}

After filling in their background information and answering questions on their attitude toward English learning, the respondents were asked to provide answers to 16 Likert questions using paper and pencil according to a 5-point Likert indicating their degree of confidence for each item. In this scale, $1=$ not at all, $2=$ barely, $3=$ somewhat, and $4=$ well. In addition, participants were given a fifth response option titled don't know, which they were instructed to choose if they had no experience with the content of the statements. The original survey was conducted in Japanese. The survey items were derived from the EIKEN can-do list (STEP, 2008). The 16 items consisted of four statements in Grade 5, Grade 4, Grade 3, and Grade Pre-2, respectively, and the statements of each EIKEN grade were composed of the four major skill areas (Appendix A).

\section{Data Analysis Methods}

The four-point responses given by the participants were coded by assigning a number to each response as follows: $1=$ not at all, $2=$ barely, $3=$ somewhat, and $4=$ well. The other option, don't know, was treated as missing data because it does not have any meaning as ordinary data. The coded data were analyzed using the Rasch-based computer software WINSTEPS (Linacre, 2004). The missing data were included in the analysis, that is, participants' responses were included as long as they responded to at least one of the 16 can-do items.

The following information was analyzed in the present study: (a) the Rasch person reliability index, (b) the item point-biserial correlations, (c) the item fit statistics, (d) the person-item threshold maps, (e) the calibrated item measures, and (f) the mean person measure at each school year and EIKEN level. The first information reported in the Rasch analysis was the reliability of separation index and person separation ratio, which indicate how distinctly students are separated by the items. The ratio is analogous to K-R20 and Cronbach's coefficient alpha in the framework of classical test theory. High separation reliability-on a 0 to 1 scale-is desirable if items are designed to differentiate low- and high-proficiency persons specifically.

The point-biserial correlation coefficients and item fit statistics were scrutinized to investigate the unidimensionality of the can-do items. Point-biserial correlation coefficients were estimated between responses for each item and others. Hence, items that have different response patterns from others should demonstrate low or negative values. Linacre (2008) claimed that point-biserial correlations should be positive, although the specific value is less important than fit statistics if the correlation is positive.

With regard to fit statistics, two types of fit indices, outfit and infit, were reported. Outfit is estimated based on the sum of squared standardized residuals of all responses including outliers; infit, on the other hand, is an information-weighted sum, 
which is less sensitive to extreme values (Bond \& Fox, 2007). Mean square values of both fit statistics are expected to be 1.0. Although there is no universal cut-off mean square range of the fit statistics, Wright, Linacre, Gustafson, and Martin-Löf (1994) provided reasonable ranges for item outfit and infit mean squares according to test types and use. The acceptable range of fit statistics for Likert scale survey items is from 0.6 to 1.4 (Wright et al., 1994), and a fit index exceeding the upper limit indicates an underfitting item.

The thresholds of each item were described in a single map that also illustrated person measures and the logit scale. By referring to the map, it is possible to see the relationship between a person's overall ability and item difficulty, which is useful for investigating item difficulty for a particular sample (Bond \& Fox, 2007). Thresholds of one standard distribution on either side of the person ability mean were analyzed to grasp the central tendency of the participants.

Finally, item and person measures calibrated by WINSTEPS were closely analyzed. Unlike item measures, person measures are not fixed, but estimated in relation to the item difficulties. A person measure is set at the point along the measurement scale where that person has an estimated 50 percent probability of endorsing the adjacent item thresholds. Depending on the sample being tested, mean person measures may be higher or lower than zero logits. Thus, when two groups with different proficiency levels take the same test, the mean logit score of high-proficiency persons will be higher than that of low-proficiency persons. Paired $t$-tests were then conducted to investigate whether or not there are statistically significant differences in item difficulty between two items belonging to distinct EIKEN grades.

\section{Results}

Below is a discussion of the results in terms of each research question.

\section{Q: Does each can-do item appear to measure a unidimensional construct?}

The person separation ratio and the reliability of separation index were 3.20 and .91 respectively which indicated that the can-do items were internally consistent and highly reliable.

Table 2 presents each can-do item's calibrated measure in a logit scale with its standard error, fit indices (infit and outfit), and point-biserial correlation coefficient. The point-biserial correlation of each item listed in the sixth column was positive and robust, ranging from 0.62 to 0.73 . With regard to item fit statistics, infit mean squares ranged from 0.87 to 1.11 , and outfit mean squares ranged from 0.87 to 1.10 . All the infit and outfit indices were within the acceptable range (from 0.6 to 1.4 ). The mean infit and outfit statistics were both close to $1.0-1.01$ and 0.99 respectively-and their standard deviations were 0.07 and 0.06 respectively. 
Table 2. Item Measure, Fit Statistics, and Point-biserial Correlation of the Can-do Items

\begin{tabular}{lccccc}
\hline Item & Measure & Error & $\begin{array}{c}\text { Infit } \\
\text { mean square }\end{array}$ & $\begin{array}{c}\text { Outfit } \\
\text { mean square }\end{array}$ & $\begin{array}{c}\text { Point-biserial } \\
\text { correlation }\end{array}$ \\
\hline Reading GP-2 & 1.29 & 0.03 & 1.01 & 1.00 & .69 \\
Reading G3 & 1.04 & 0.03 & 0.92 & 0.97 & .69 \\
Reading G4 & 0.83 & 0.04 & 0.87 & 0.87 & .73 \\
Reading G5 & -1.65 & 0.04 & 1.05 & 0.94 & .67 \\
Listening GP-2 & 1.20 & 0.04 & 0.97 & 1.05 & .71 \\
Listening G3 & 0.26 & 0.04 & 0.91 & 0.91 & .72 \\
Listening G4 & -1.31 & 0.04 & 1.10 & 0.95 & .69 \\
Listening G5 & -1.92 & 0.04 & 1.11 & 1.10 & .64 \\
Speaking GP-2 & 0.81 & 0.03 & 1.05 & 1.06 & .69 \\
Speaking G3 & -0.23 & 0.04 & 0.98 & 0.95 & .70 \\
Speaking G4 & -1.25 & 0.04 & 1.04 & 0.99 & .67 \\
Speaking G5 & -1.88 & 0.04 & 1.08 & 0.97 & .62 \\
Writing GP-2 & 2.23 & 0.04 & 0.95 & 0.93 & .71 \\
Writing G3 & 1.38 & 0.03 & 1.05 & 1.02 & .73 \\
Writing G4 & 0.52 & 0.03 & 1.02 & 1.06 & .69 \\
Writing G5 & -1.33 & 0.04 & 1.10 & 1.01 & .67 \\
\hline Mean & 0.00 & 0.04 & 1.01 & 0.99 & \\
SD & 1.32 & 0.00 & 0.07 & 0.06 & \\
\hline
\end{tabular}

Note. GP $-2=$ EIKEN Grade Pre-2; G3 = EIKEN Grade 3; G4 = EIKEN Grade 4; G5 = EIKEN Grade 5.

\section{Q: Is the difficulty of the can-do items appropriate for the target students?}

Figure 1 displays the alignment between the participants and the multiple thresholds of the can-do items. The numbers written vertically on the left side (from 6 to -6 ) are in logits, and the distribution of the participants is illustrated next to the scale (each \# represents 12 participants and each dot represents a number of participants less than 12). The symbols $M, S$, and $T$ denote the person/item mean, one standard deviation $(S D)$ from the mean, and two $S D$ s from the mean respectively. A person who is listed at a higher position on the map chose higher valued options for the questionnaire items and demonstrated a higher level of confidence. The right of the continuum shows the thresholds of each can-do item. For example, "Writing GP-2.3" in Figure 1 indicates that persons with the same logits are $50 \%$ likely to choose either $2=$ barely or $3=$ somewhat. Above that label, the most probable option is 3 . An item that is listed at a higher position was endorsed less by the participants and therefore demonstrated a higher level of difficulty. 


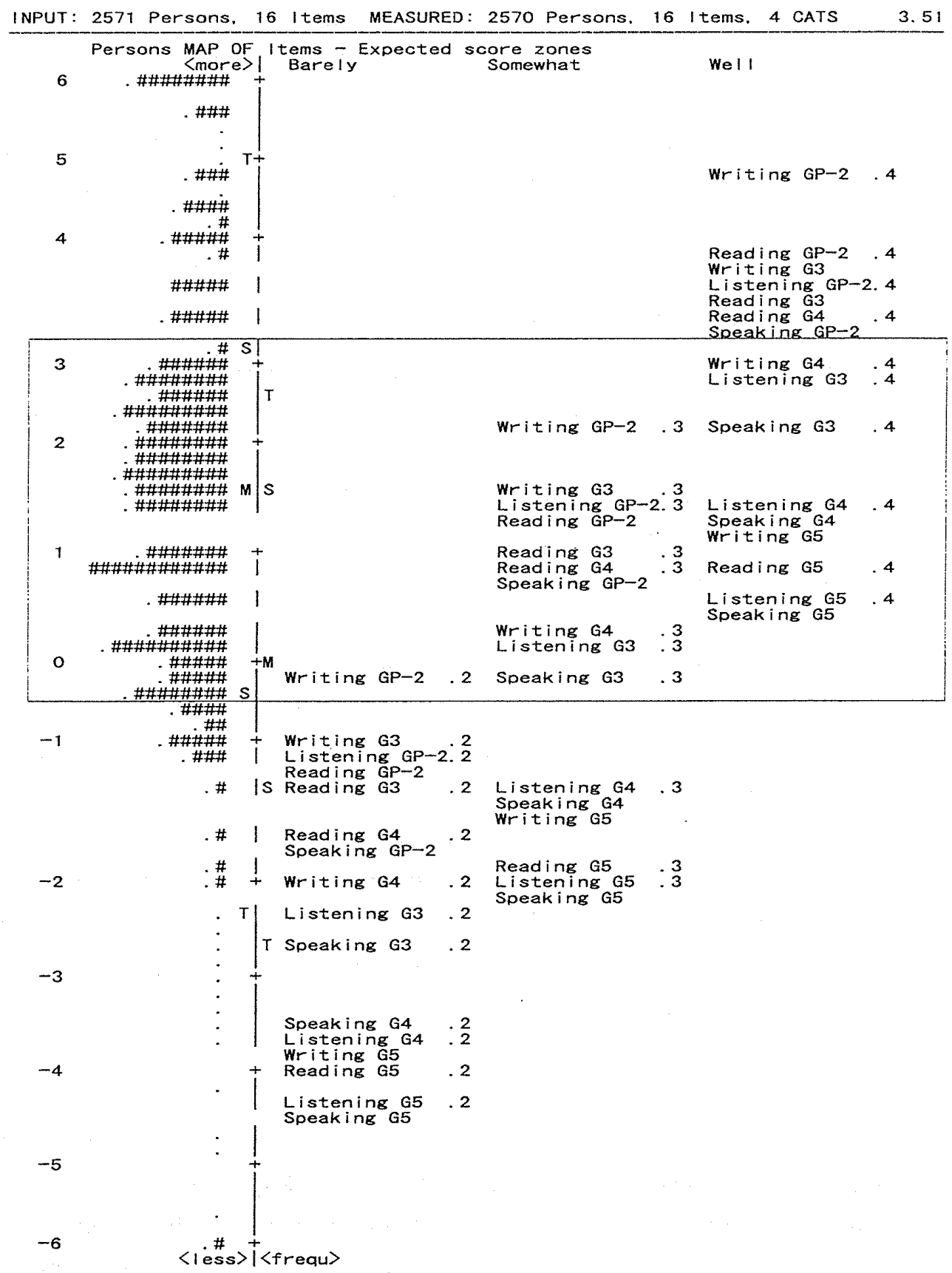

Figure 1. Person-item threshold map $(\mathrm{N}=2,751)$

The item map shows that the distribution of participants was heavily skewed toward the lower measure. In particular, there were a relatively few participants below one $S D$ from the mean (14.5\% of the participants). Compared to those with low logit scores, more participants were located above one $S D$ from the mean $(19.0 \%)$, indicating that their responses to all of the can-do items were likely to be $4=$ well. Furthermore, some participants' logits were estimated at more than $6(3.7 \%)$, which indicated a ceiling effect. Comparing the position of the person and item means, it is clear that the person mean was located higher than the item mean. 
Next, the thresholds of the participants who were located within one $S D$ from the mean were examined in order to grasp the central tendency of the sample $(66.5 \%$ of the participants). The thresholds in the surrounded area in Figure 1 show the response tendency of the average participants. More specifically, it is possible to classify the can-do items into four clusters in the order of item difficulty with the most difficult first.

1. One item with thresholds of " 2 " and " 3 ": Writing GP-2.

2. Six items with a threshold of " 3 ": Listening GP-2, Speaking GP-2, Reading GP-2, Reading G3, Writing G3, and Reading G4.

3. Three items with thresholds of "3" and "4": Listening G3, Speaking G3, and Writing G4.

4. Six items with a threshold of "4": Listening G4, Speaking G4, Listening G5, Speaking G5, Writing G5, and Reading G5.

Similarly, responses of EIKEN grade holders-Grade 5, Grade 4, Grade 3, and Grade Pre-2-were analyzed separately (each person-item threshold map is presented in Appendix B). Overall, the distributions of the participants were heavily skewed toward the lower measure. The result of each EIKEN grade shows that a large number of participants reached Threshold 4 for all corresponding can-do items. For example, students who had EIKEN Grade 5 tended to endorse a high category ( 3 or 4) for all Grade 5 can-do items, suggesting that they were at least moderately confident with those items. Furthermore, those maps indicate that the participants tended to choose high categories for higher EIKEN grade items as well, because the thresholds of those items around the person mean were three and four (regarded as positive responses). For example, students with EIKEN Grade 5 tended to choose high levels options for Grade 4 items.

\section{Q: Do items belonging to the different EIKEN grades (from Grade 5 to Grade Pre-2) reflect students' self-reported confidence levels appropriately?}

Table 2 shows the calibrated measures and standard errors of the 16 can-do items. Items high in the logit scale were items that the participants did not endorse highly, that is, items for which the students' confidence level was low. Figure 2 presents the calibrated item measures and the changes in difficulty according to the four EIKEN levels (Grade 5 to Grade Pre-2). As shown in Figure 2, the can-do items for higher EIKEN grades demonstrated higher item measures than lower grades without exception.

Table 3 presents the differences in item measures of each skill area between adjacent EIKEN grades. Paired $t$-tests were carried out to scrutinize the statistical significance of the gaps. The result shows all the items demonstrated statistically significant differences at $p<.01$ (one-tailed) between each EIKEN grade. In other words, the can-do items belonging to higher EIKEN grades were distinct from items 
belonging to lower grades. However, differences in item difficulty largely varied according to skill areas and EIKEN grades since the gaps ranged from 0.21 to 2.48 . The maximum and minimum gaps were both observed in reading-between Grade 5 and Grade 4 (maximum) and between Grade 4 and Grade 3 (minimum).

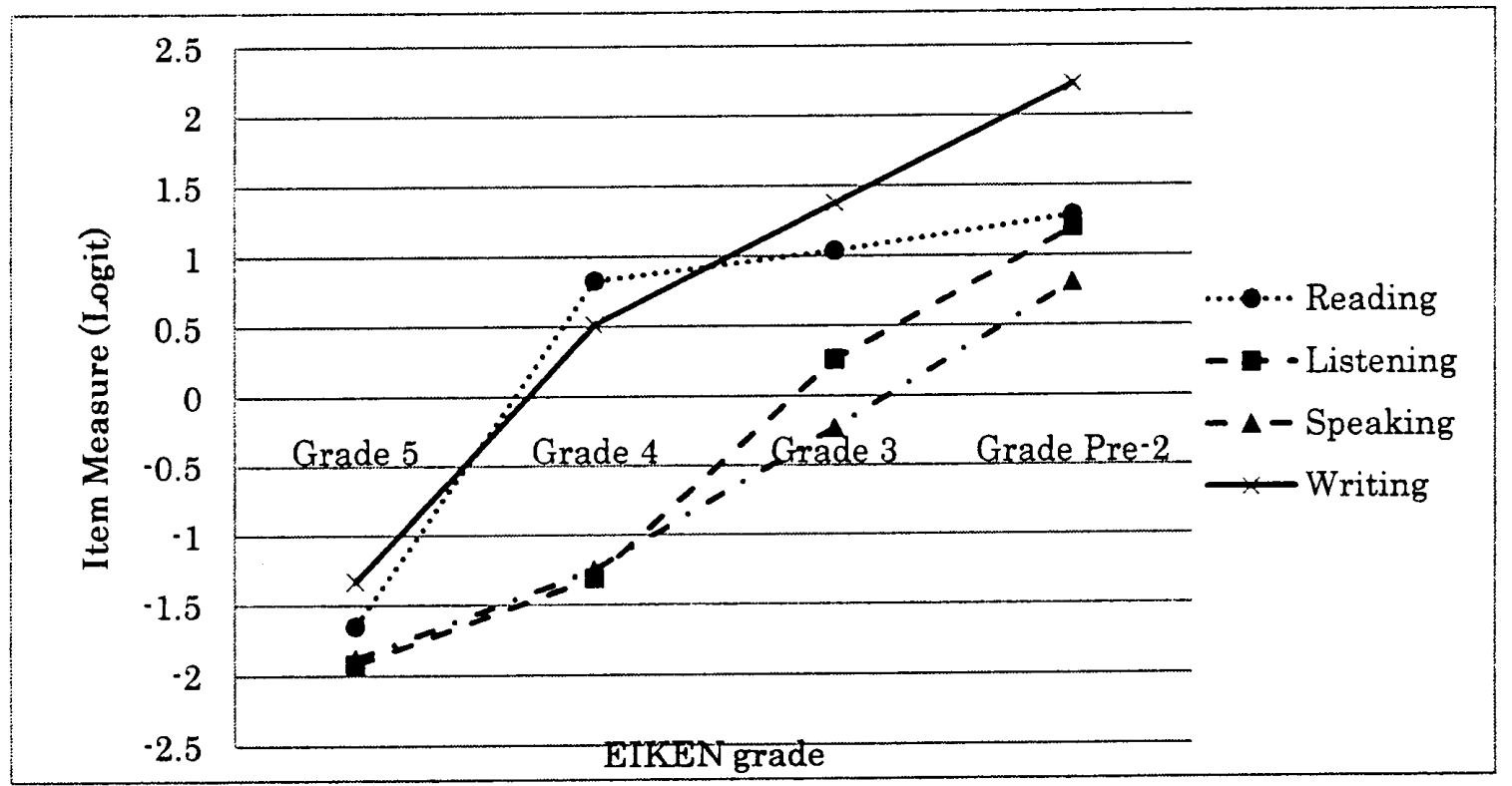

Figure 2. Item measures of 16 can-do items for four different EIKEN grades

Table 3. Paired Differences in Item Measures between Adjacent EIKEN Grades

\begin{tabular}{lccc}
\hline Skill Area & Grade 5-Grade 4 & Grade 4-Grade 3 & Grade 3-Grade Pre-2 \\
\hline Reading & $2.48^{* *}$ & $0.21^{* *}$ & $0.25^{* *}$ \\
Listening & $0.61^{* *}$ & $1.57^{* *}$ & $0.94^{* *}$ \\
Speaking & $0.63^{* *}$ & $1.02^{* *}$ & $1.04^{* *}$ \\
Writing & $1.85^{* *}$ & $0.86^{* *}$ & $0.85^{* *}$ \\
\hline
\end{tabular}

Note. ${ }^{* *} p<.01$.

Q: Do the EIKEN can-do items properly reflect the differing confidence levels of students with different proficiency levels?

Table 4 presents the mean person measures of students in each school year, the $S D$, and the standard error. As the results show, the mean measures increased linearly as the students' school year rose (1.35, 1.50, and 2.12 logits, respectively). This suggests that students in higher school years tended to select higher can-do options than those in lower school years. Moreover, all the mean measures were far above the mean item measure, indicating that they were inclined to demonstrate high confidence in the 16 can-do items overall. 
Table 4. The Mean and Error of Person Measures and the SD of Each School Year

\begin{tabular}{|c|c|c|c|c|c|c|}
\hline & \multicolumn{2}{|c|}{$\begin{array}{c}1^{\text {st }} \text { yr. students } \\
(N=694)\end{array}$} & \multicolumn{2}{|c|}{$\begin{array}{c}2^{\text {nd }} \text { yr. students } \\
(N=1,365)\end{array}$} & \multicolumn{2}{|c|}{$\begin{array}{c}3^{\text {rd }} \text { yr. students } \\
(N=493)\end{array}$} \\
\hline & Measure & Error & Measure & Error & Measure & Error \\
\hline Mean & 1.35 & 0.52 & 1.50 & 0.57 & 2.12 & 0.58 \\
\hline$S D$ & 1.87 & 0.23 & 2.28 & 0.35 & 2.17 & 0.34 \\
\hline
\end{tabular}

Table 5 presents the mean person measures of students with each EIKEN grade (from Grade 5 to Grade Pre-2). The ascending mean measures (1.92, 2.41, 3.53, and 5.43 logits) indicate that the students with higher EIKEN grades tended to show more confidence with the 16 can-do items.

Table 5. The Mean and Error of Person Measures and the SD of Each EIKEN Level

\begin{tabular}{|c|c|c|c|c|c|c|c|c|}
\hline & \multicolumn{2}{|c|}{$\begin{array}{c}\text { Grade } 5 \\
(N=210)\end{array}$} & \multicolumn{2}{|c|}{$\begin{array}{c}\text { Grade } 4 \\
(N=474)\end{array}$} & \multicolumn{2}{|c|}{$\begin{array}{c}\text { Grade } 3 \\
(N=126)\end{array}$} & \multicolumn{2}{|c|}{$\begin{array}{l}\text { Grade Pre-2 } \\
\quad(N=36)\end{array}$} \\
\hline & Measure & Error & Measure & Error & Measure & Error & Measure & Error \\
\hline Mean & 1.92 & 0.51 & 2.41 & 0.54 & 3.53 & 0.62 & 5.43 & 0.77 \\
\hline$S D$ & 1.63 & 0.13 & 1.68 & 0.15 & 1.61 & 0.17 & 1.88 & 0.19 \\
\hline
\end{tabular}

\section{Discussion}

Below is an interpretation of each of the four research questions.

\section{Q: Does each can-do item appear to measure a unidimensional construct?}

The 16 EIKEN can-do items being investigated showed a single underlying pattern of scores; therefore, each of them is likely to measure a unidimensional construct. The point-biserial correlation values and the item fit statistics indicated the unidimensionality when those values were compared with the standards proposed by Wright et al. (1994). The range of fit indices that were narrowly grouped around 1.0 imply that the data elicited from the participants were close to the response patterns expected by the Rasch model, and there was no item whose response patterns were too unpredictable or haphazard. In other words, a person demonstrating high overall confidence in the EIKEN can-do items tended to respond to all individual items at a high level. However, it should be noted that while fit mean square statistics were relatively robust for different sample sizes, they get closer to 1.0 if the number in the sample increases (Bond \& Fox, 2007). There were a large number of participants in the present study, and this might be one of the causes for the fit indices to be close to 1.0 and the extremely small $S D$. 


\section{Q: Is the difficulty of the can-do items appropriate for the target students?}

It might be that the 16 can-do items taken from four EIKEN grades were perceived to be too easy for the junior high school students since the item map in Figure 1 showed a heavily skewed distribution and thus suggested an inadequate alignment between the items and the students. In fact, $19 \%$ of the participants (located higher than one $S D$ from the mean in Figure 1) were likely to choose a high category for Grade Pre-2 items, even though only $2 \%$ of the participants had EIKEN grades higher than Grade 3. Moreover, although EIKEN Grade 3 is considered an official benchmark for junior high school graduates in Japan (STEP, 2008), the results suggest that the junior high school students with average ability tended to have moderate confidence with regard to those items.

The responses of different EIKEN grade holders showed that students with a particular EIKEN grade tended to be confident with all corresponding can-do items. This is a positive result because the EIKEN can-do list provides descriptions of what students who passed a certain EIKEN grade can do outside testing situations (STEP, 2008). However, the result also showed that the students are somewhat confident with can-do items one grade higher than their EIKEN grade. For example, the EIKEN Grade 5 holders tended to endorse a high category for can-do items from Grades 4 and 3 (especially speaking and listening). In other words, students with EIKEN Grade 5 might believe that they can perform activities in Grades 4 and 3 to some degree. These results indicate that the can-do items might be seen as unchallenging by students overall.

It should be noted that item difficulty differed depending on the channel (audio or visual) to a great extent. In general, audio-channel items were perceived to be easier than visual-channel items. Their logits and thresholds showed that the speaking and listening items in a certain EIKEN grade (e.g., Grade 4) are similar in difficulty to the reading and writing items in a lower EIKEN grade (e.g., Grade 5). In other words, for example, the students tended to show almost the same level of confidence in Speaking G4, Listening G4, Reading G5, and Writing G5.

Since the primary purpose of the EIKEN can-do list is "to help test users gain a better understanding of the levels of language ability targeted by the EIKEN tests" (STEP, 2008, p. 4), learners are likely to think that items belonging to higher grades in the list are subgoals for learning English. As far as students' motivation is concerned, the low item difficulty may be a cause for concern because setting proximal subgoals is one of the necessary conditions for maintaining students' motivation (Dörnyei, 2001). If students perceive higher EIKEN grade items to be unchallenging, they may not be motivated to improve their English proficiency. Therefore, it is suggested that users of the can-do list need to be cautious about its use in light of low item difficulty. More specifically, it may be necessary for students to regard speaking and listening items that are two grades higher than their current EIKEN level as appropriate subgoals. 
Q: Do items belonging to the different EIKEN grades (from Grade 5 to Grade Pre-2) reflect students' self-reported confidence levels appropriately?

The can-do items from the higher EIKEN grades demonstrated significantly higher item measures as shown in Figure 2, which supports the classification of items into distinct grades according to item difficulty. However, it is important to note that the differences greatly varied between each adjacent EIKEN grade. In particular, the gap between Reading G5 and G4 was the largest, and the gap between Reading G4 and G3 was the smallest. It can be considered that Reading G4 was perceived to be too difficult; in fact, this item was regarded as more difficult than Speaking GP-2. A possible reason for the high item difficulty might be the students' unfamiliarity with the written genre of Reading G4 (short letters or e-mails). For example, textbooks used in junior high schools generally contain only a few units dealing with letters or e-mails, whereas familiar topics related to daily life (Reading G3) and simple expository texts (Reading GP-2) are discussed in many units. ${ }^{3}$ This lack of experience is considered to affect their judgment, since an item that is less related to the target students' experience and situation is likely to be judged less accurately (Oscarson, 1997, p. 183; Ross, 1998, p. 16).

\section{Q: Do the EIKEN can-do items properly reflect the differing confidence levels of students with different proficiency levels?}

Finally, the results indicated that the students in higher school years showed more confidence with the 16 can-do statements. Since those students had spent more time learning English, the can-do items reflected the difference in the students' English learning experience. Furthermore, the students with higher EIKEN grades tended to show more confidence in the items compared with those with lower EIKEN grades. This result suggests that the can-do items properly reflect the students' English proficiency levels, that is, the more proficient students become, the more confident they become. Although it has been reported that Japanese people tend to be modest and that high level L2 learners assess their ability more critically (Matsuno, 2009), it can be said that the 16 can-do items successfully differentiated the participants based on language proficiency and learning experience.

\section{Conclusion}

The present study examined 16 EIKEN can-do items on the basis of the responses of 2,571 junior high school students, using the Rasch rating scale model. The results show that the 16 items were highly reliable and fit the Rasch expectation, which indicates that each of them meaningfully contributed to a unique underlying construct. As a whole, the classification of items according to the EIKEN grade levels appeared to be warranted since the item difficulty increased consistently as the EIKEN grades rose. 
The items were also correlated with students' English proficiency levels and the length of time they had spent learning English. However, users of the can-do items must be careful about their use in light of the following two results. First, the 16 items were relatively easy for junior high school students, although the items were taken from a wide range-Grade 5 to Grade Pre-2. In particular, even speaking and listening items in high EIKEN grades were perceived to be relatively unchallenging. Second, Reading G4 was perceived to be more difficult than it was supposed to be.

Contrary to the more common methods used in validation studies of SA measures, the present study validated the can-do items through Rasch measurement without relying on external criteria that must be validated before comparison. The Rasch analysis made it possible to carry out in-depth item-level analysis.

Although the present study provided evidence for the validity of 16 EIKEN can-do items, it would be too ambitious to generalize the results for all the items in the EIKEN can-do list. In particular, the present study examined only one item taken from each EIKEN grade (from Grade 5 to Grade Pre-2) and each skill area. Thus, it is still necessary to confirm the validity of the remaining can-do items from the same EIKEN grades and skill areas in order to validate the whole can-do list. Moreover, the 16 investigated items were taken from only four EIKEN grades-from the elementary level to the intermediate level. The present study did not investigate any items from the three more advanced grades (i.e., Grade 2, Grade Pre-1, and Grade 1), which must also be validated by further research.

\section{Acknowledgements}

The present study is a part of a large-scale project on early English language education by SOLIFIC in collaboration with JIEM. I am thankful to the junior high school students who responded to the questionnaire. I am deeply grateful to Yoshinori Watanabe for his helpful comments on an early draft of this paper. My thanks also go to two anonymous reviewers whose suggestions have improved this article.

\section{Notes}

1. The students were not asked to supply gender data in the questionnaire.

2. The complete questionnaire form and an overall research report on the project will be published (SOLIFIC \& JIEM, in press).

3. The following textbooks for junior high school students authorized by the MEXT were examined: New Horizon (Tokyo Shoseki), Sunshine (Kairyudo), Total English (Gakko Tosho), New Crown (Sanseido), One World (Kyoiku Shuppan), and Columbus 21 (Mitsumura Tosho). 


\section{References}

Alderson, J. C. (2005). Diagnosing foreign language proficiency: The interface between learning and assessment. London, England: Continuum.

Alderson, J. C., Clapham, C., \& Wall, D. (1995). Language test construction and evaluation. Cambridge, England: Cambridge University Press.

Bailey, K. (1998). Learning about language assessment: Dilemmas, decisions, and directions. Cambridge, MA: Heinle and Heinle.

Blanche, P., \& Merino, B. J. (1989). Self-assessment of foreign-language skills: Implications for teachers and researchers. Language Learning, 39, 313-340.

Bond, T. G., \& Fox, C. M. (2007). Applying the Rasch model: Fundamental measurement in the human sciences (2nd ed.). Mahwah, $\mathrm{NJ}$ : Lawrence Erlbaum Associates.

Brantmeier, C. (2005). Advanced L2 learners and reading placement: Self-assessment, CBT, and subsequent performance. System, 34, 15-35.

Brantmeier, C., \& Vanderplank, R. (2008). Descriptive and criterion-referenced self-assessment with L2 readers. System, 36, 456-477.

Brown, H. D., \& Abeywickrama, P. (2010). Language assessment: Principles and classroom practice (2nd ed.). White Plains, NY: Pearson Education.

Brown, J. D., \& Hudson, T. (2002). Criterion-referenced language testing. Cambridge, England: Cambridge University Press.

Davies, A., Brown, A., Elder, C., Hill, K., Lumley, T., \& McNamara, T. (1999). Studies in language testing 7: Dictionary of language testing. Cambridge, England: Cambridge University Press.

Dörnyei, Z. (2001). Teaching and researching motivation. Essex, England: Pearson Education.

Dunlea, J. (2009). The EIKEN Can-do List: Improving feedback for an English proficiency test in Japan. In L. Taylor \& C. J. Weir (Eds.), Studies in language testing 31: Language testing matters (pp. 245-262). Cambridge, England: Cambridge University Press.

Falchikov, N., \& Boud, D. (1989). Student self-assessment in higher education: A meta-analysis. Review of Educational Research, 59, 395-430.

Harmer, J. (2007). The practice of English language teaching (4th ed.). Essex, England: Pearson Education.

Linacre, J. M. (2004). Winsteps (Version 3.51) [Computer software]. Chicago, IL: MESA Press.

Linacre, J. M. (2008). A user's guide to Winsteps Ministep Rasch-model computer programs. Chicago, IL: Winsteps.com.

Matsuno, S. (2009). Self-, peer-, and teacher-assessments in Japanese university EFL writing classrooms. Language Testing, 26, 75-100. 
Mok, M. M. C. (2004). Validation of scores from self-learning scales for primary students using true-score and Rasch measurement methods. Journal of Applied Measurement, 5, 258-286.

Oscarson, M. (1997). Self-assessment of foreign and second language proficiency. In C. Clapham \& D. Corson (Eds.), Language testing and assessment (pp. 175-187). The Netherlands: Kluwer Academic Publishers.

Peirce, B. N., Swain, M., \& Hart, D. (1993). Self-assessment, French immersion, and locus of control. Applied Linguistics, 14, 25-42.

Ross, S. (1998). Self-assessment in second language testing: A meta-analysis and analysis of experiential factors. Language Testing, 15, 1-20.

Society for Testing English Proficiency. (2008). The EIKEN can-do list. Tokyo, Japan: The Society for Testing English Proficiency.

Sophia Linguistic Institute for International Communication \& Japan Institute for Educational Measurement. (in press). Sokieigo gakusyuno chugakueigo gakusyueno eikyo: Jyoui can-do ishikichosa [An effect of early English learning on English learning in junior high schools: A study of learners' attitudes and confidence]. Tokyo, Japan: Printboy.

Sullivan, K., \& Hall, C. (1997). Introducing students to self-assessment. Assessment \& Evaluation in Higher Education, 22, 289-306.

Takemura, M. (2008). Eiken can-do risutoniyoru writing ginonikansuru datoseino kensho [Investigation of the validity of the EIKEN Can-do List for writing ability: using the lists of Grade Pre-2 and Grade 3]. STEP Bulletin, 20, 251-261.

Usuta, Y. (2009). Eiken can-do risutono supikingubunyaniokeru can-do koumokuno datouseikensho [Investigation of the validity of can-do items for speaking skills in the EIKEN Can-do List]. STEP Bulletin, 21, 262-273.

Weaver, C. (2005). Using the Rasch model to develop a measure of second language learners' willingness to communicate within a language classroom. Journal of Applied Measurement, 6, 396-415.

Wright, B. D., Linacre, J. M., Gustafson, J-E., \& Martin-Löf, P. (1994). Reasonable mean-square fit values. Rasch Measurement Transactions, 8, 370. 


\section{Appendix A: 16 EIKEN Can-do Items}

\begin{tabular}{|c|c|}
\hline \multicolumn{2}{|c|}{ Reading } \\
\hline Pre-2 & $\begin{array}{l}\text { Can understand simple expository texts (e.g. learning materials introducing the lifestyles in } \\
\text { foreign countries and foreign cultures). }\end{array}$ \\
\hline 3 & Can understand texts about familiar topics related to everyday life (e.g. sports, music). \\
\hline 4 & $\begin{array}{l}\text { Can understand short letters and e-mails (e.g. introducing the writer's family, about } \\
\text { memories of a trip). }\end{array}$ \\
\hline 5 & $\begin{array}{l}\text { Can understand basic sentences describing familiar activities from everyday life (e.g. "I play } \\
\text { tennis every day."). }\end{array}$ \\
\hline \multicolumn{2}{|c|}{ Listening } \\
\hline Pre-2 & $\begin{array}{l}\text { Can understand a speaker on the telephone, provided the content is simple (e.g. agreeing } \\
\text { when to meet, taking short messages). }\end{array}$ \\
\hline 3 & $\begin{array}{l}\text { Can understand simple announcements, provided that the speaker speaks slowly and/or } \\
\text { repeats sections (e.g. meeting place, arrival and departure times for transportation). }\end{array}$ \\
\hline 4 & $\begin{array}{l}\text { Can understand the meaning of simple instructions (e.g. "Open your textbook." / "Close the } \\
\text { door, please."). }\end{array}$ \\
\hline 5 & Can understand everyday greetings (e.g. "How are you?" / "Nice to meet you."). \\
\hline \multicolumn{2}{|c|}{ Speaking } \\
\hline Pre-2 & $\begin{array}{l}\text { Can express his/her ideas about things that he/she is interested in (e.g. sports he/she likes, } \\
\text { things related to his/her hobbies). }\end{array}$ \\
\hline 3 & $\begin{array}{l}\text { Can talk briefly about something that he/she is interested in (e.g. his/her hobbies, club } \\
\text { activities). }\end{array}$ \\
\hline 4 & Can give a simple self-introduction (e.g. name, where he/she lives, about his/her family). \\
\hline 5 & Can apologize and express thanks (e.g. "I'm sorry." / "Thank you."). \\
\hline \multicolumn{2}{|c|}{ Writing } \\
\hline Pre-2 & Can write short letters and e-mails (e.g. a simple letter to a friend or pen pal). \\
\hline 3 & Can write a short diary entry (from one to three sentences). \\
\hline 4 & Can write sentences joining clauses with conjunctions (e.g. and, but, so, when, because). \\
\hline 5 & Can write familiar words from everyday life (e.g. dog, eat, happy). \\
\hline
\end{tabular}




\section{Appendix B: Person-item Threshold Maps of Students with Each EIKEN Grade}

EIKEN Grade 5 holders $(\mathrm{N}=210)$

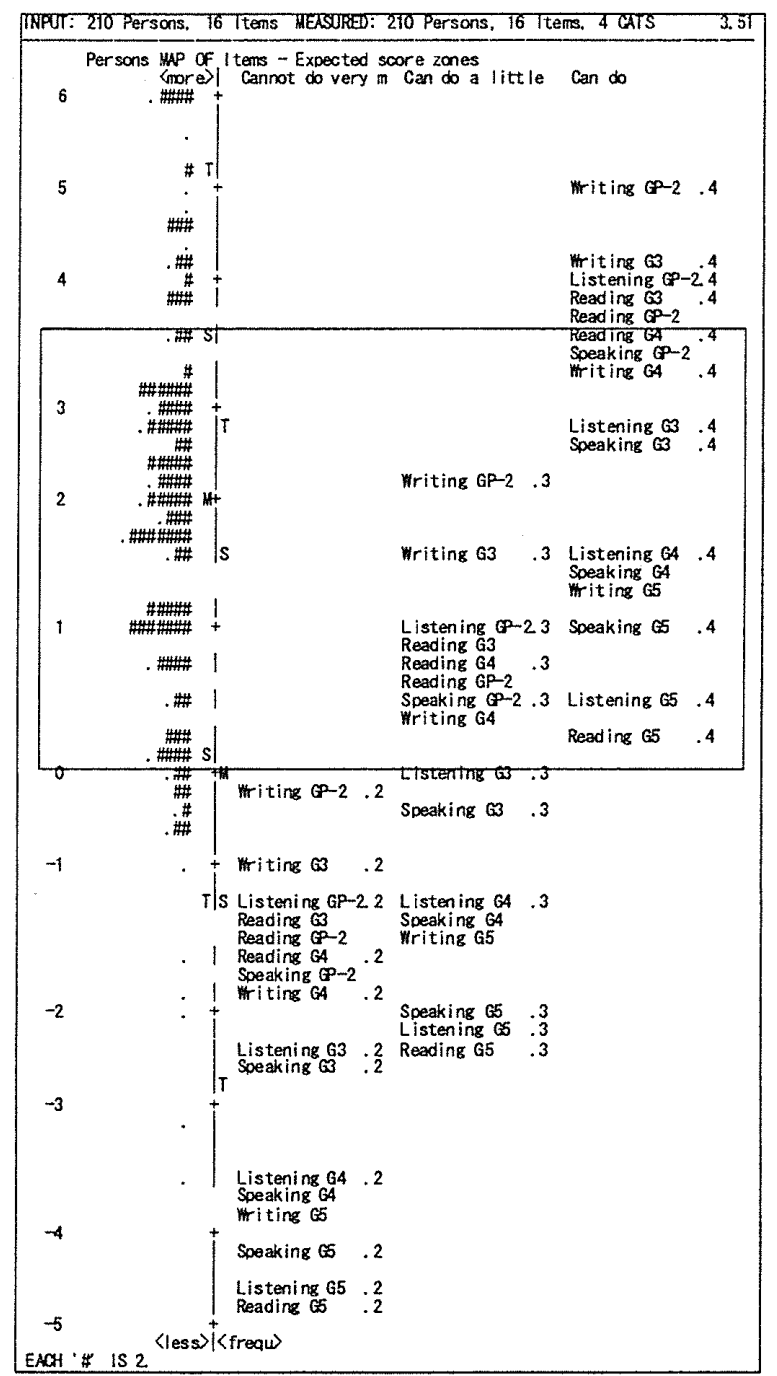

EIKEN Grade 4 holders $(N=474)$

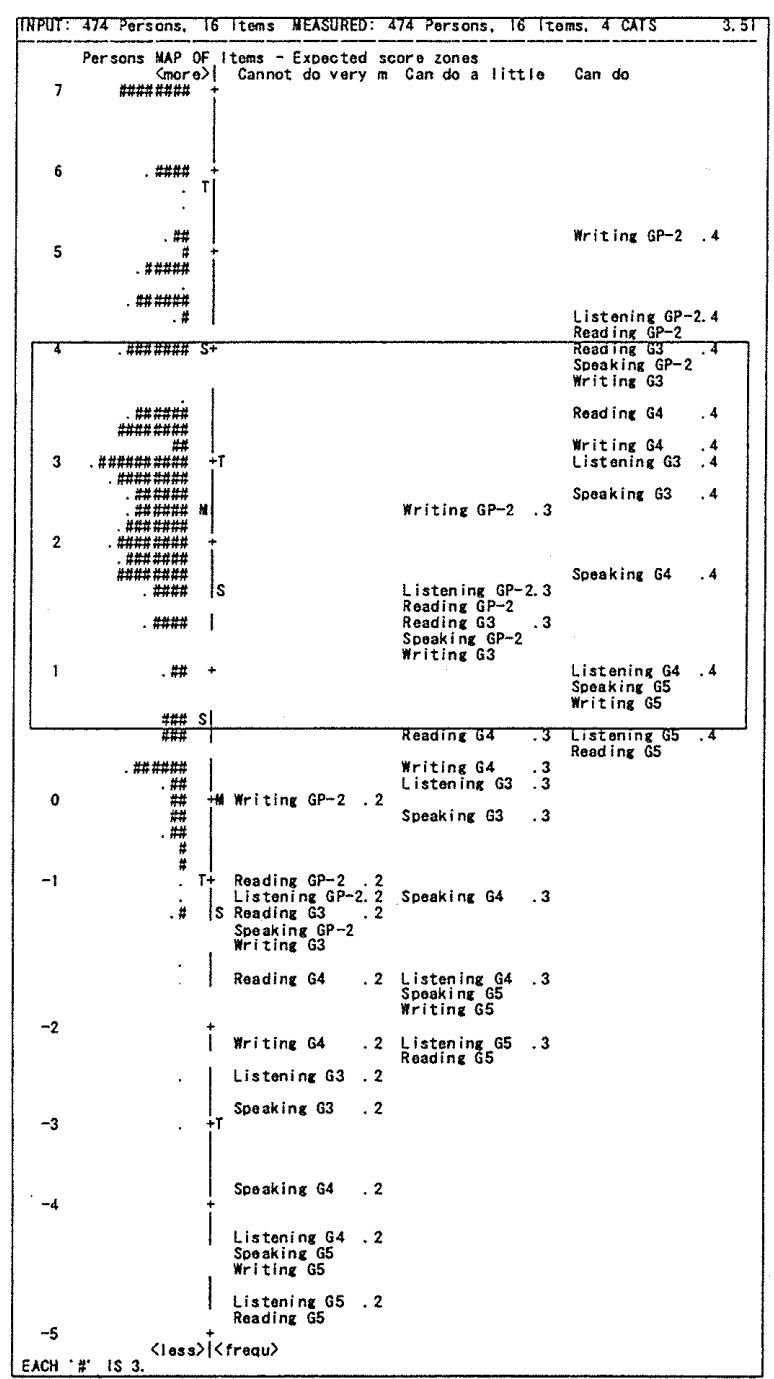


EIKEN Grade 3 holders ( $N=126)$

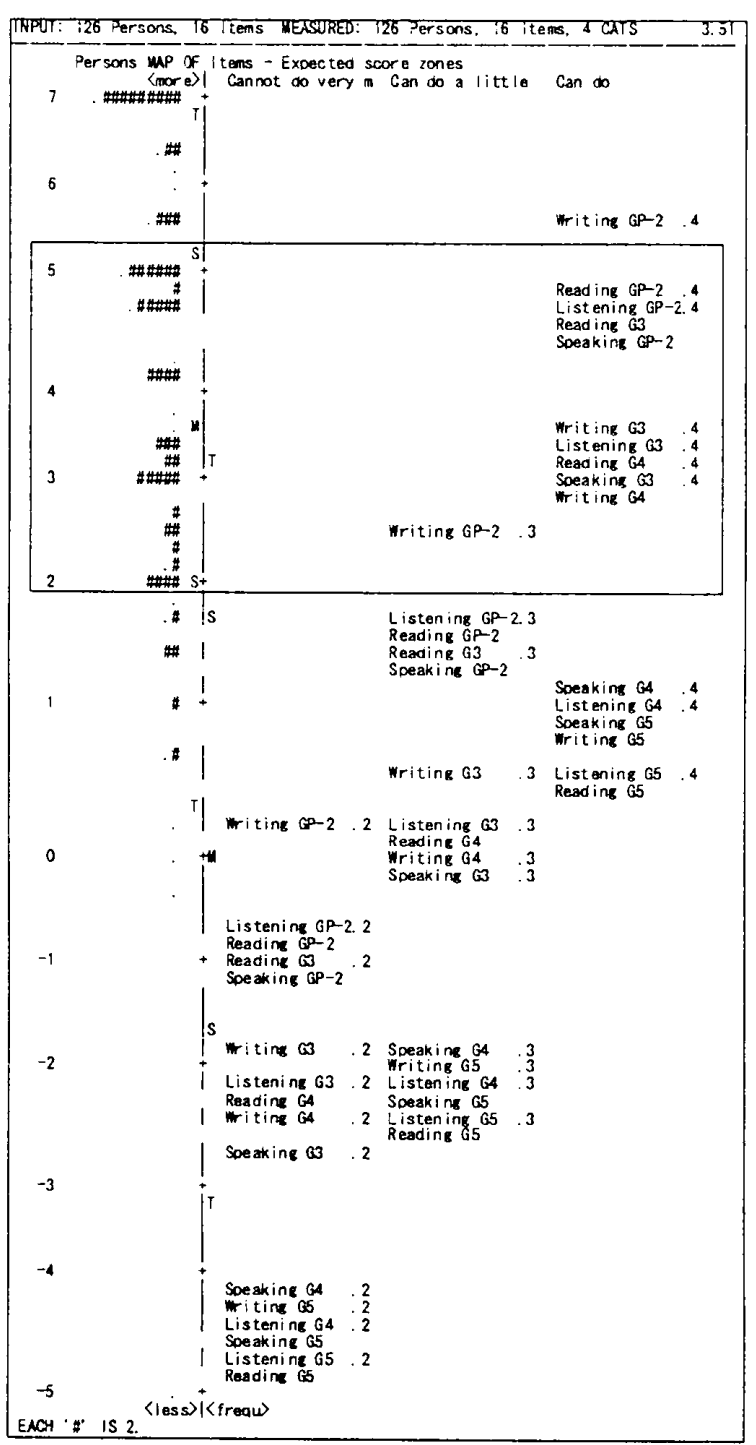

EIKEN Grade Pre-2 holders ( $\mathrm{N}=36$ )

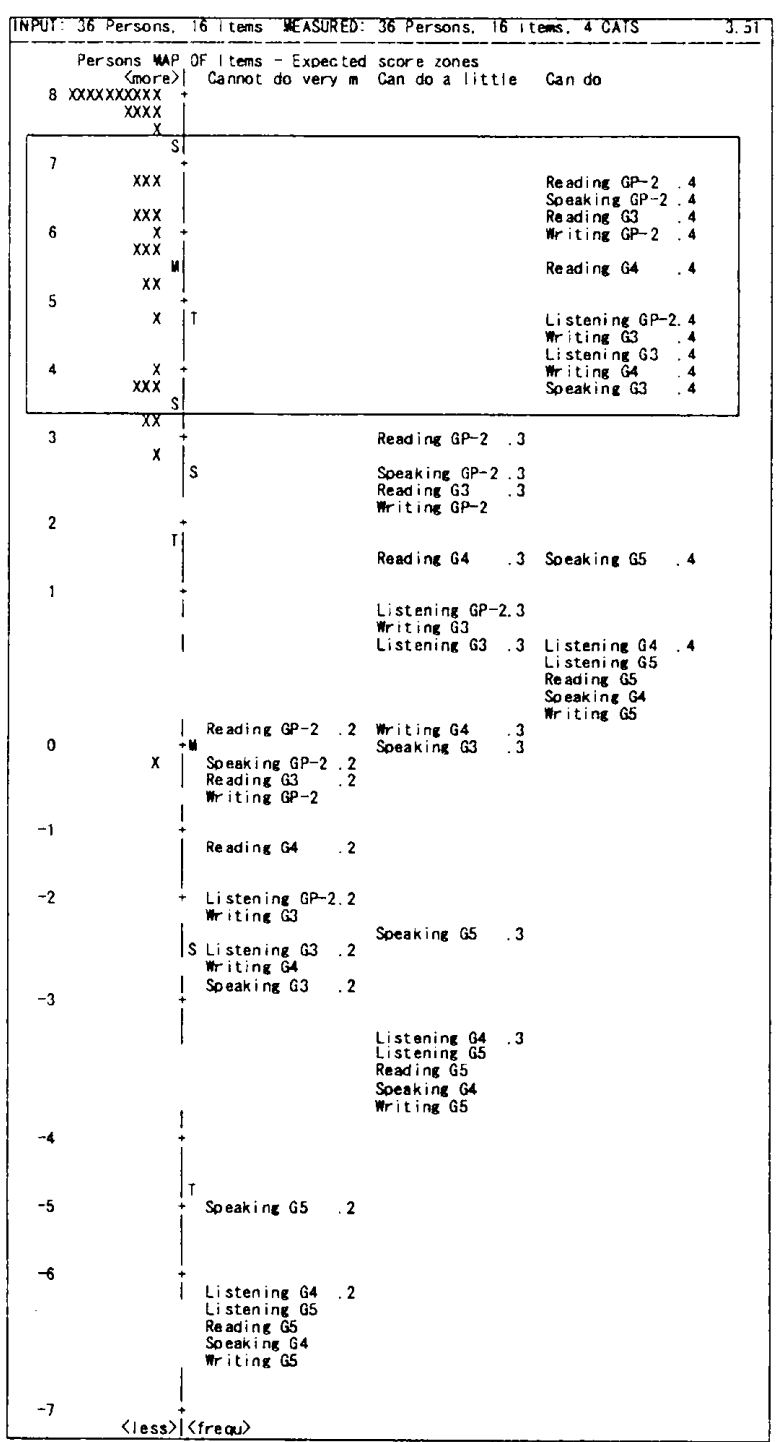

